\title{
Stabilisation chimique de deux sols gonflants de la région d'Oran
}

A. HACHICHI

S. BOUROKBA

Institut de qénie civil

Université des sciences et de la technologie

BP 1505, El M'naouar Oran, Algérie hachichi_geotech@yahoo.fr

\section{J.-M. FLEUREAU}

Laboratoire de mécanique des sols, structures \& matériaux

CNRS UMR 8579

et École centrale Paris

Grande Voie des Vignes 92295 Châtenay-Malabry France jean-marie.fleureau@ecp.fr
Cette étude a été réalisée sur des sols provenant de deux sites situés à proximité de la ville d'Oran (nord-ouest de l'Algérie) oú l'on rencontre de nombreux problèmes de fissuration de bâtiments et de soulèvement de fond de fouilles. Les sols ont été caractérisés du point de vue minéralogique et du point de vue géotechnique. Ces essais ont été complétés par cles mesures directes de gonflement à l'eau. Dans une seconde étape, l'étude a porté sur l'effet de différentes solutions salines minérales ou organiques à différentes concentrations sur l'amplitude du gonflement. Les sels utilisés ont conduit à des réductions très importantes du gonflement pouvant atteindre $80 \%$. Afin d'estimer les modifications à long terme apportées par les fluides hydratants, les limites de liquidité de plasticité et de retrait des argiles stabilisées, ont été mesurées. On note une bonne corrélation entre l'effet des sels sur le gonflement libre et la diminution de plasticité, les meilleurs résultats étant obtenus en présence de $\mathrm{KCl}$ et les moins bons, avec $\mathrm{CaCl}_{2}$.

Mots-clés: argiles gonflantes, gonflement, pression de gonflement, stabilisation chimique, Algérie.
The research was carried out on soils from two sites in the vicinity of the town of Oran (Northwest of Algeria) where many problems of fissures in buildings and heave of foundations are encountered. The soils were characterized from both mineralogical and geotechnical points of view. Swelling in soil was also investigated. In a second step, the effect of different saline mineral or organic solutions at different concentrations on swelling was studied. Significant decreases in swelling up to $80 \%$ were observed with some salts. In order to assess the long-term effect of the treatment, the liquid, plastic and shrinkage limits of the stabilized soils were measured. General agreement is observed between the effect of salts on swelling and the decrease in plasticity of the clays, the best results being obtained with $\mathrm{KCl}$ and the worse with $\mathrm{CaCl}_{2}$.

Key words : expansive soils, swelling, swelling pressure, chemical treatment, Algeria. 


\section{NOTATIONS}

w : Teneur en eau massique $(\%)$

$w_{i}$ : Teneur en eau initiale $(\%)$

$w_{\text {nat }}$ : Teneur en eau naturelle $(\%)$

$w_{L}$ : Limite de liquidité

$\mathrm{w}_{\mathrm{p}}$ : Limite de plasticité

$I_{p} \quad$ : Indice de plasticité

$\mathrm{w}_{\text {St. }}$ : Limite de retrait (\%)

$I_{S 1 .} \quad$ : Indice de retrait

e : Indice des vides

$\gamma_{\mathrm{s}} \quad$ : Poids volumique des grains solides $\left(\mathrm{kN} / \mathrm{m}^{3}\right)$

$\gamma_{\mathrm{d}}$ : Poids volumique sec du sol $\left(\mathrm{kN} / \mathrm{m}^{3}\right)$

$\mathrm{P}_{2} \quad$ : Pourcentage de grains de diamètre inférieur à $2 \mu \mathrm{m}$

$\mathrm{D}_{60} \quad$ : Diamètre correspondant à $60 \%$ de passants ( $\mu \mathrm{m})$

$A_{c} \quad$ : Activitè $\left[=I_{\rho} / P_{2}\right]$

$V_{\mathrm{B}}$ : Valeur de bleu

$\mathrm{SS}_{\mathrm{T}}$ : Surface spécifique totale $\left(\mathrm{m}^{2} / \mathrm{g}\right)$

G : Gonflement libre (en \%)

$\mathrm{P}_{\mathrm{g}} \quad$ : Pression de gonflement $(\mathrm{kPa})$

$\mathrm{t}_{100}$ : temps correspondant à la fin du gonflement primaire (mn)

\section{Introduction}

De nombreux problèmes de fissuration rencontrés dans les canalisations enterrées, les trottoirs, les bâtiments et les routes ne résultent pas d'un chargement excessif du sol d'assise mais du gonflement ou du retrait de celui-ci. En Algérie, plusieurs cas de désordres très préjudiciables, liés au gonflement, ont été signalés sur des ouvrages dans plusieurs régions: la raffinerie pétrolière de In Amenas au sud-est du pays (Ameur, 1989 ; Derrich et al., 1998 ; Hachichi et Fleureau, 1999; Lamara et al., 2005), I'hôpital de N'Gaous et la ligne de chemin de fer Ramdane Djamel (nord-est), I'hôpital de Sidi Chahmi et la briqueterie de Mers-el Kébir situés au nord-ouest du pays à proximité de la ville d'Oran. Les sols des deux derniers sites cités cidessus sont à l'origine de fissurations très préjudiciables dans les bâtiments de l'hôpital (Fig. 1) et de soulèvements non négligeables des fonds de foulle ayant entraîné la fissuration des longrines (Fig. 2).

L'étude présentée a comme objectif de caractériser les sols sur lesquels ces deux ouvrages sont construits et d'étudier leur stabilisation chimique au laboratoire par des sels et des polymères.

De nombreuses recherches ont été menées pour tester l'efficacité de diverses solutions salines susceptibles de réduire le gonflement des argiles (Chenevert et O'Brien, 1973, cités par Chaouch, 2001; Azzouz, 1983 ; Suratman, 1985; Basma et al., 1998; Hachichi et Fleureau, 1999; Bourokba et al., 2001 ; Hachichi et al., 2002; Ho Young et al., 2001). L'efficacité d'un sel dans la réduction du gonflement d'une argile peut être liée à la capacité d'échange, à la valence, à la nature et la taille des cations qui jouent un rôle majeur dans les substitutions ioniques qui se produisent à l'intérieur des feuillets argileux. La probabilité d'échange des cations augmente dans l'ordre suivant:

$$
\mathrm{Li}^{+}<\mathrm{Na}^{+}<\mathrm{K}^{+}<\mathrm{NH}_{4}^{+}<\mathrm{Mg}^{2+}<\mathrm{Ca}^{2+}<\mathrm{Al}^{3+}
$$

Les polymères, qui sont des sels organiques, sont attirés par la surface des argiles quand ils portent des charges positives et, dans certaines conditions de $\mathrm{pH}$, par les extrémités des feuillets quand les charges qu'ils portent sont négatives. La grande taille de ces molécules permet une sorte d'encapsulage qui limite l'hydratation ultérieure de l'argile. L'interaction des polymères avec

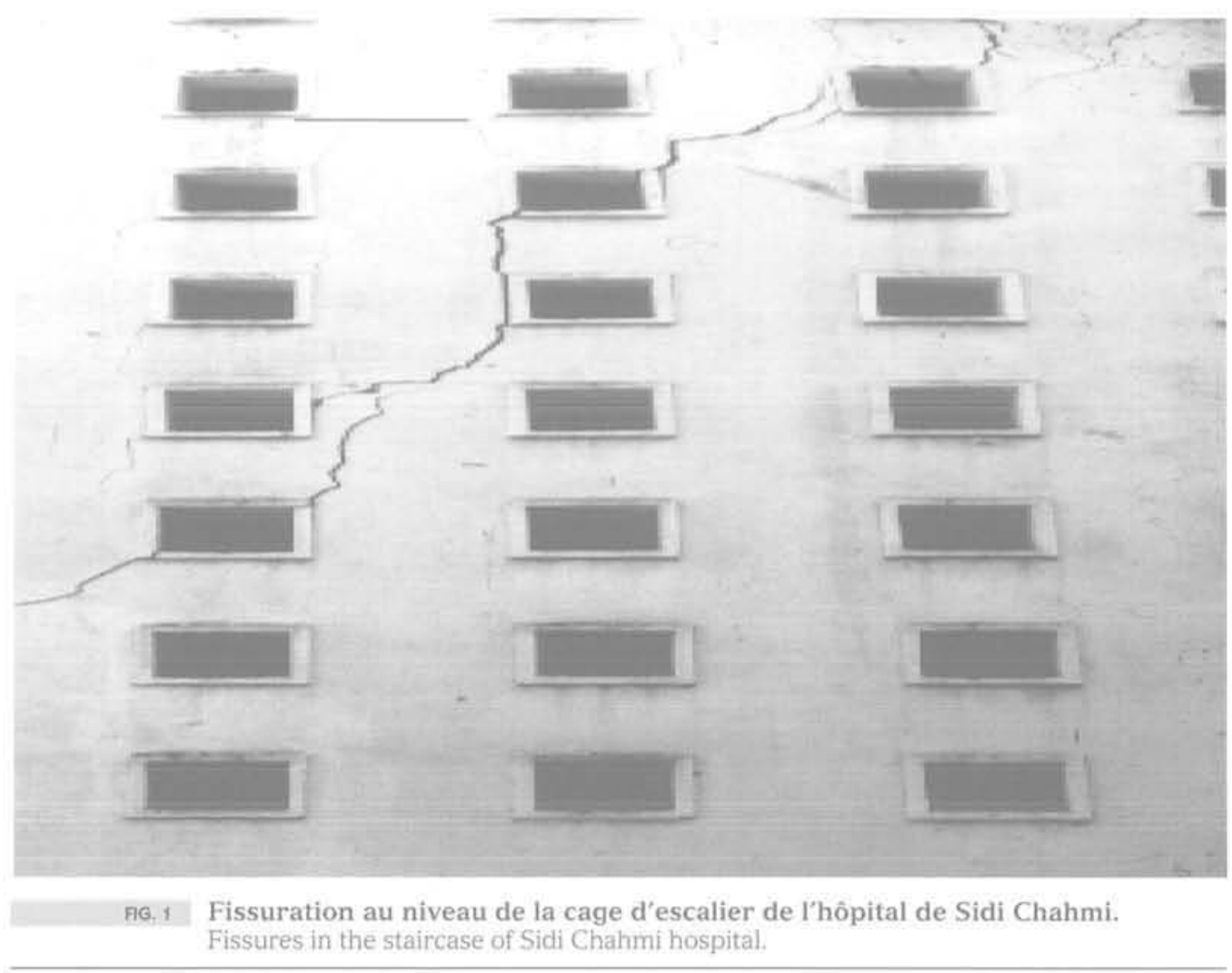




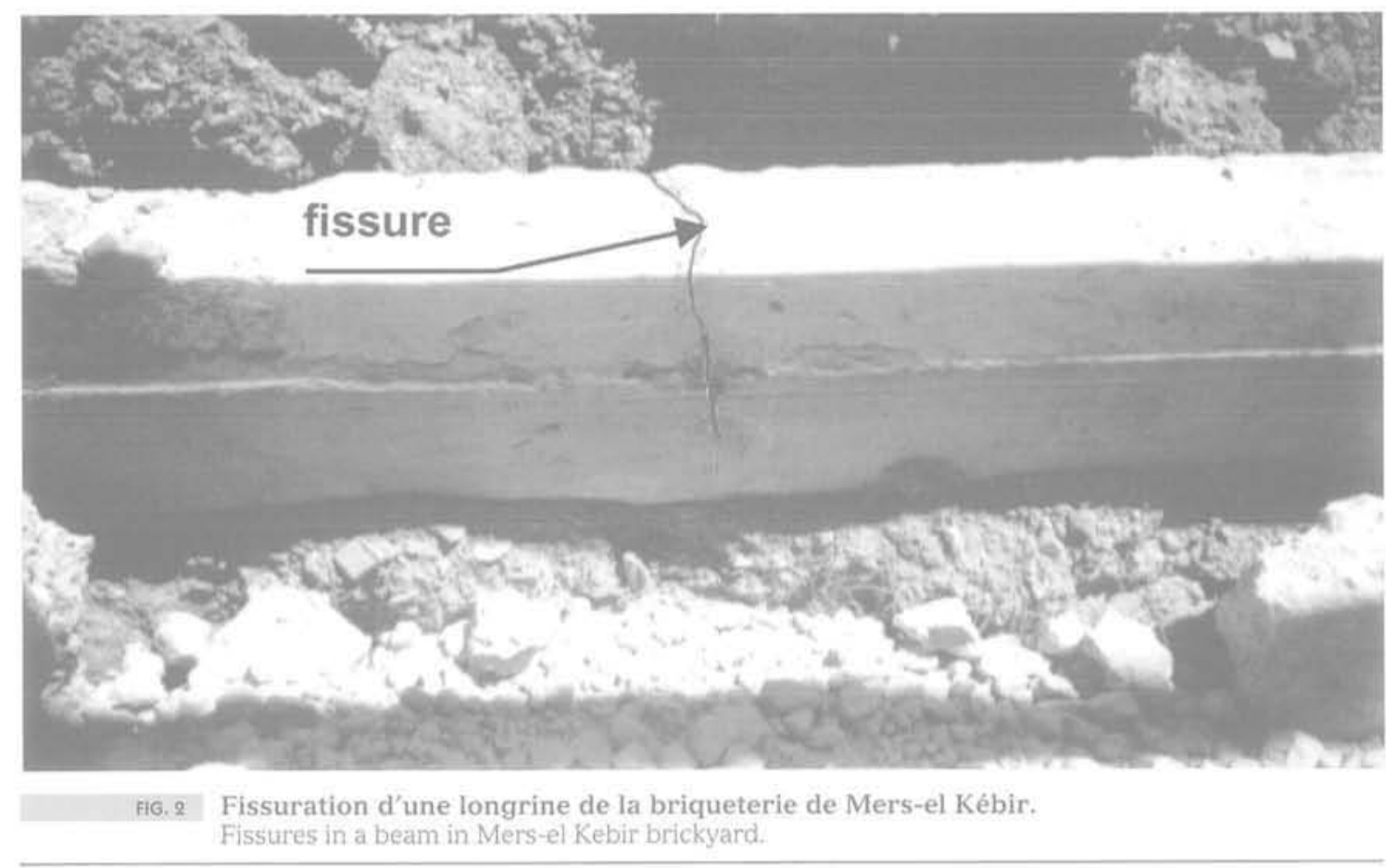

les argiles dépend du type d'argile, de la dimension des grains et de la nature des cations échangeables (Breen, 1999). Il a été montré que l'association d'un sel et d'un polymère pouvait être plus efficace vis-à-vis de la stabilisation que le simple emploi de l'un ou de l'autre composant (Azzouz, 1983). La dernière partie de l'étude est par conséquent consacrée à l'effet de quelques mélanges sel-polymère sur le gonflement des deux argiles.

\section{2}

\section{Localisation et géologie des sites}

Les matériaux de Sidi Chahmi et Mers-el Kébir ont fait l'objet de recherches antérieures (Hachichi et Fleureau, 1999; Bourokba et al., 2001).

Le premier site se situe à environ $6 \mathrm{~km}$ à l'est de la ville d'Oran et à $2 \mathrm{~km}$ au nord-est du village de Sidi
Chahmi. Il est constitué de deux couches superposées, la couche supérieure composée d'argile lacustre du quaternaire avec des passées sableuses et calcaires, la couche inférieure formée de marne plus au moins sableuse du Pliocène.

Le site de la briqueterie de Mers-el Kébir, situé au nord-ouest d'Oran, est constitué par des marnes grises et vertes. Ce sont des formations sédimentaires marines lagunaires vers le sommet, connues sous l'appellation de marnes bleues du Miocène supérieur. Leur épaisseur peut parfois dépasser 300 mètres.

\section{3}

\section{Analyses minéralogique et chimique}

Une analyse diffractométrique aux rayons $\mathrm{X}$ à été menée pour déterminer les minéraux constituant les

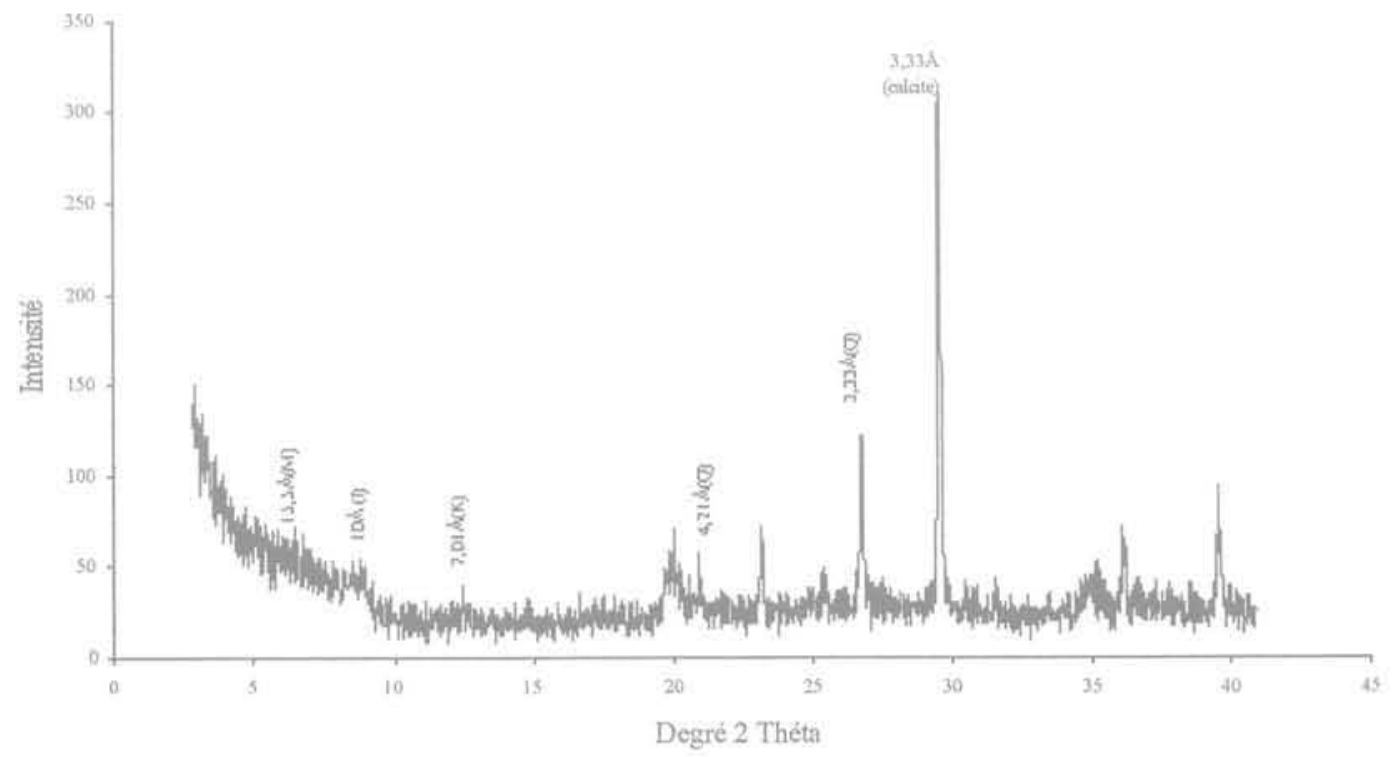

FG. 3 Analyse diffractométrique de I'argile de Sidi Chahmi. X-ray diffractometer of Sidi Chahmi clay, 


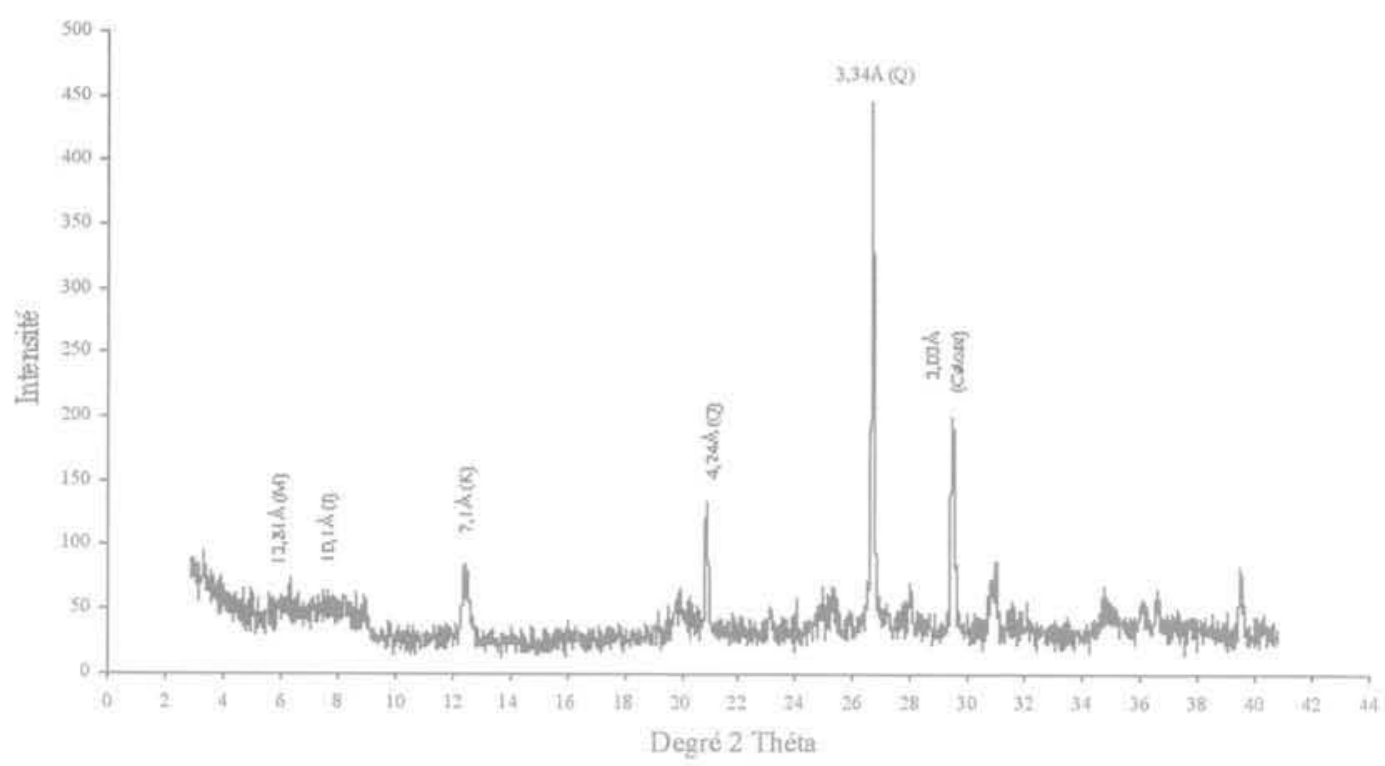

FIG. 4 Analyse diffractométrique de l'argile de Mers-el Kébir. X-ray diffractometer of Mers-el Kebir clay.

sols étudiés; les résultats sont présentés sur les figures 3 et 4 . Cette analyse montre que les deux argiles sont formées de kaolinite, d'illite, d'interstratifiés illitemontmorillonite, de quartz et de calcite. L'analyse chimique des deux matériaux a été réalisée par fluorescence $\mathrm{X}$. Les résultats, résumés dans le tableau I, montrent que les deux argiles sont des alumino-silicates avec un rapport silicium/aluminium de l'ordre de 3,2 pour l'argile de Sidi Chahmi et de 3,5 pour l'argile de Mers-el Kébir. Le pourcentage de $\mathrm{Fe}_{2} \mathrm{O}_{3}$ et de $\mathrm{CaO}$ confirme la présence de goethite et de calcite dans les deux argiles, le taux de potassium varie de 2 à $3 \%$. La capacité d'échange cationique de ces argiles est de $30 \mathrm{meq} / \mathrm{g}$ pour l'argile de Sidi Chahmi et de $26 \mathrm{meq} / \mathrm{g}$ pour l'argile de Mers-el Kébir. Ces valeurs sont relativement faibles par rapport à celles des montmorillonites pures (qui peuvent dépasser $100 \mathrm{meq} / \mathrm{g}$ ), ce qui est conforme à l'analyse minéralogique.

\section{Identification géotechnique des matériaux}

Les résultats des essais d'identification sont rassemblés dans le tableau II. D'après les classifications LCPC et USCS, l'argile de Sidi Chahmi est très plastique Ap $(\mathrm{CH})$ et l'argile de Mers-el Kébir est peu plastique At $(\mathrm{CL})$. Leur activité $(\mathrm{Ac}=\mathrm{lp} / \%<2 \mu \mathrm{m})$ est élevée à très élevée. La surface spécifique totale est déduite de l'essai au bleu de méthylène par la formule de Tran Ngoc Lan (1977). Les valeurs obtenues pour les
2 argiles (374 et $429 \mathrm{~m}^{2} / \mathrm{g}$, respectivement) sont élevées mais correspondent aux valeurs basses des montmorillonites (entre 300 et $800 \mathrm{~m}^{2} / \mathrm{g}$ ).

D'après la classification de Williams et Donaldson (1980) indiquée sur la figure 5, l'argile de Mers-el Kébir a un potentiel de gonflement élevé et celle de Sidi Chahmi, très élevé.

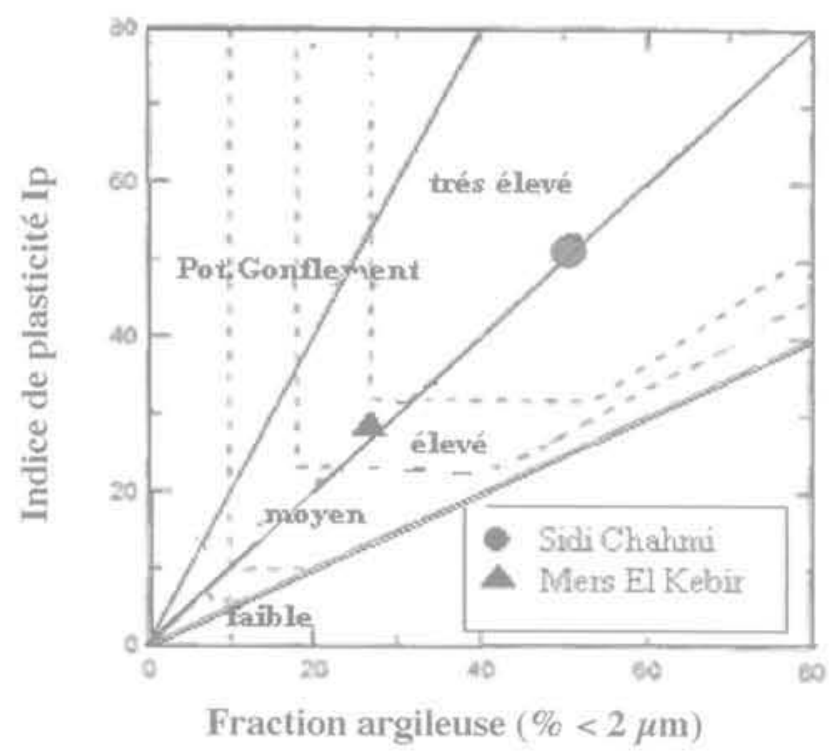

FIG.5 Classification des potentiels de gonflement d'après Williams et Donaldson (1980). Classification of swelling potentials, from Williams \& Donaldson (1980).

TABLEAVI Résultats des analyses chimiques par fluorescence $\mathrm{X}$. Results of the chemical analyses by $\mathrm{X}$ fluorescence.

\begin{tabular}{|c|c|c|c|c|c|c|c|c|c|c|}
\hline & $\begin{array}{l}\mathrm{SiO}_{2} \\
(\%)\end{array}$ & $\begin{array}{l}\mathrm{TO}_{2} \\
(\%)\end{array}$ & $\begin{array}{c}\mathrm{AlO}_{2} \mathrm{O} \\
(\%)\end{array}$ & $\begin{array}{c}\mathrm{FeO}_{3} \\
(\%)\end{array}$ & $\begin{array}{c}\mathrm{CaO} \\
(\%)\end{array}$ & $\begin{array}{c}\mathrm{MgO}) \\
(\%)\end{array}$ & $\begin{array}{l}\mathrm{BaO} \\
(\%)\end{array}$ & $\begin{array}{c}\mathrm{Na}, \mathrm{O} \\
(\%)\end{array}$ & $\begin{array}{l}K_{2} O \\
(\%)\end{array}$ & $\begin{array}{c}\mathrm{MnO} \\
(96)\end{array}$ \\
\hline Sidi Chahmi & 56,65 & 0,15 & 17,81 & 10,10 & 6,40 & 5,15 & 0,09 & 0,35 & 3,11 & 0,08 \\
\hline Mers-el Kébir & 59,42 & 0,36 & 16,89 & 10,04 & 5,38 & 5,35 & 0.10 & 0,33 & 1,95 & 0,08 \\
\hline
\end{tabular}


TABLEAU II. Résultats des essais d'identification.

Results of the identification tests.

\begin{tabular}{|c|c|c|c|c|c|c|c|c|c|c|c|c|c|c|}
\hline & $\frac{Z}{(m)}$ & $\begin{array}{l}w_{\text {nat }} \\
(\%)\end{array}$ & $\frac{\gamma_{6}}{\left(\mathrm{kN}^{2} / \mathrm{m}^{3}\right)}$ & $\frac{\gamma_{5}}{\left(\mathrm{kN} / \mathrm{m}^{3}\right)}$ & $\begin{array}{l}w_{1} \\
(\%)\end{array}$ & $\begin{array}{l}w_{p} \\
(\%)\end{array}$ & $\begin{array}{l}W_{\text {sL }} \\
(\%)\end{array}$ & Ip & $\frac{I_{s L}}{(\%)}$ & $\frac{<2 \mu m}{(\%)}$ & $A_{C}$ & $V_{B}$ & $\begin{array}{l}\mathrm{Ss}_{\mathrm{I}} \\
\left(\mathrm{m}^{2} / \mathrm{g}\right)\end{array}$ & $\begin{array}{c}\mathrm{CaCO}_{3} \\
(\%)\end{array}$ \\
\hline $\begin{array}{l}\text { Sidi Chahmi } \\
\text { Mers-el Kébir }\end{array}$ & $4,2-4,55$ & $\overline{17}, 1$ & $\overline{16.7}$ & $\begin{array}{l}26,6 \\
26,7\end{array}$ & $\begin{array}{l}73,4 \\
47,5\end{array}$ & $\begin{array}{l}23,0 \\
21,5\end{array}$ & $\begin{array}{l}7,91 \\
11.7\end{array}$ & $\begin{array}{c}50,4 \\
29\end{array}$ & $\begin{array}{l}65,2 \\
34,8\end{array}$ & $\begin{array}{l}52 \\
27\end{array}$ & $\begin{array}{c}0,96 \\
1,1\end{array}$ & $\begin{array}{l}17.8 \\
20,5\end{array}$ & $\begin{array}{l}374 \\
429\end{array}$ & $\begin{array}{l}18,2 \\
17,8\end{array}$ \\
\hline
\end{tabular}

\section{5}

\section{Mesure du gonflement libre à l'œdomètre} 5.1

\section{Méthode de mesure}

Le gonflement libre est mesuré a l'œedomètre sur un échantillon soumis au seul poids du piston, mis en contact avec un réservoir d'eau à charge nulle. La variation de hauteur du piston est mesurée en fonction du temps jusqu'à ce qu'elle se stabilise. La courbe de gonflement (Fig. 6) présente deux parties que l'on peut analyser, par analogie avec le phénomène de consolidation. comme des phases de gonflement primaire et secondaire. La valeur finale du gonflement, après stabilisation, permet de calculer la variation relative de volume de l'échantillon, notée $\mathrm{G}$, que l'on exprime en pourcentage. La même procédure est utilisée pour étudier le gonflement des échantillons en présence de solutions salines ou de solutions de polymères. La réduction du gonflement final $\Delta G / G_{0}$ exprimée en pourcents est la différence entre le gonflement à l'eau $G_{0}$ et avec les solutions salines $G$, rapportée au gonflement final en présence d'eau $G_{0}$.

\section{2}

\section{Préparation des échantillons}

Les essais ont été effectués sur des échantillons remaniés. Après avoir broyé finement le sol, la poudre est mélangée à l'eau de façon homogène puis compactée sous presse à vitesse lente $(1 \mathrm{~mm} / \mathrm{min})$ dans un moule de $50 \mathrm{~mm}$ de diamètre et $100 \mathrm{~mm}$ de hauteur. La teneur en eau des échantillons est fixée à $20 \%$ et leur densité sèche, à $17 \mathrm{kN} / \mathrm{m}^{3}$ (teneur en eau et densité sèche proches de celles des échantillons intacts). Les échantillons sont ensuite prélevés dans des anneaux oedométriques de $10 \mathrm{~mm}$ de hauteur, puis séchés à l'étuve à $60^{\circ} \mathrm{C}$ jusqu'à teneur en eau nulle.

\section{3}

\section{Choix des sels pour la réduction du gonflement}

Les sels minéraux utilisés pour la stabilisation sont le chlorure de potassium ( $\mathrm{KCl}$ ), le chlorure de calcium $\left(\mathrm{CaCl}_{2}\right)$, le chlorure de sodium $(\mathrm{NaCl})$, le sulfate de sodium $\left(\mathrm{Na}_{2} \mathrm{SO}_{4}\right)$ et le chlorure d'ammonium $\left(\mathrm{NH}_{4} \mathrm{Cl}\right)$ à différentes concentrations $(0,05,0,5,1$ et 2 moles/litre).

Les polymères utilisés à différentes concentrations sont au nombre de quatre :

- le carboxyl méthyl cellulose de faible viscosité : CMC LV (à 2, 4, 6 et 8 g/1),

- le carboxyl méthyl cellulose traité de faible viscosité : CMC LVT (à 2, 4, 6 et $8 \mathrm{~g} / 1$ ),

- le carboxyl méthyl cellulose de haute viscosité : CMC HVT (à 1,3 et $4,5 \mathrm{~g} / 1$ ),

- I'hydroxyl éthyl cellulose : NATRASOL HEC (à 1, 2 et $4 \mathrm{~g} / 1$ ).

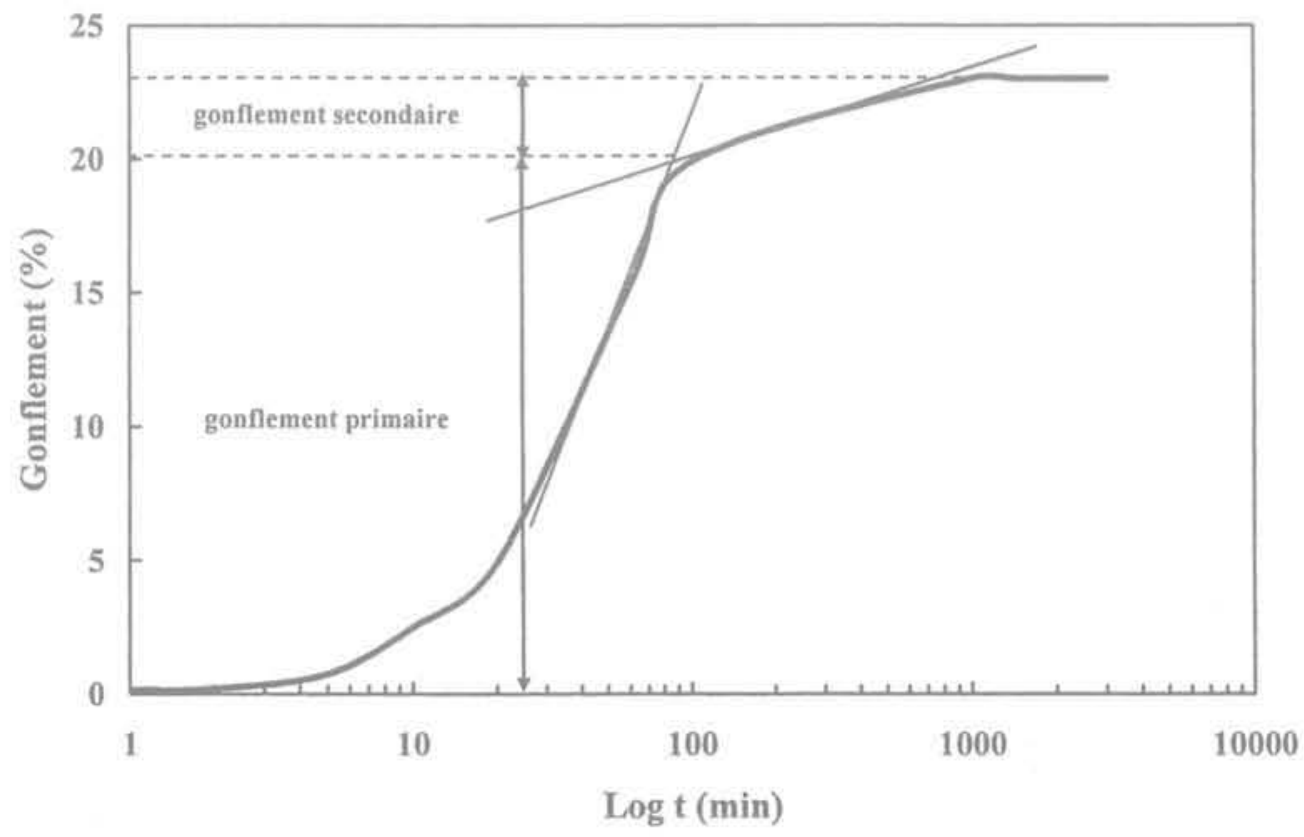

FG.6 Courbe de gonflement en fonction du temps. Swelling curve versus time. 
6

\section{Résultats obtenus}

\section{Réduction du gonflement par les sels minéraux}

La figure 7 représente les courbes de gonflement des argiles de Sidi Chahmi et de Mers-el Kébir en présence des sels qui ont donné le plus grand pourcentage de réduction. Nous remarquons que la réduction est surtout observée pour de fortes concentrations en chlorure de potassium et en chlorure de calcium (66 \% et $52 \%$ ) pour l'argile de Sidi Chahmi ( $44 \%$ et $48 \%$ ) pour celle de Mersel Kébir. Par contre, le chlorure d'ammoniumest moyennement efficace à forte concentration pour l'argile de Sidi Chahmi (29\%) et pour l'argile de Mers-el Kébir (38\%).
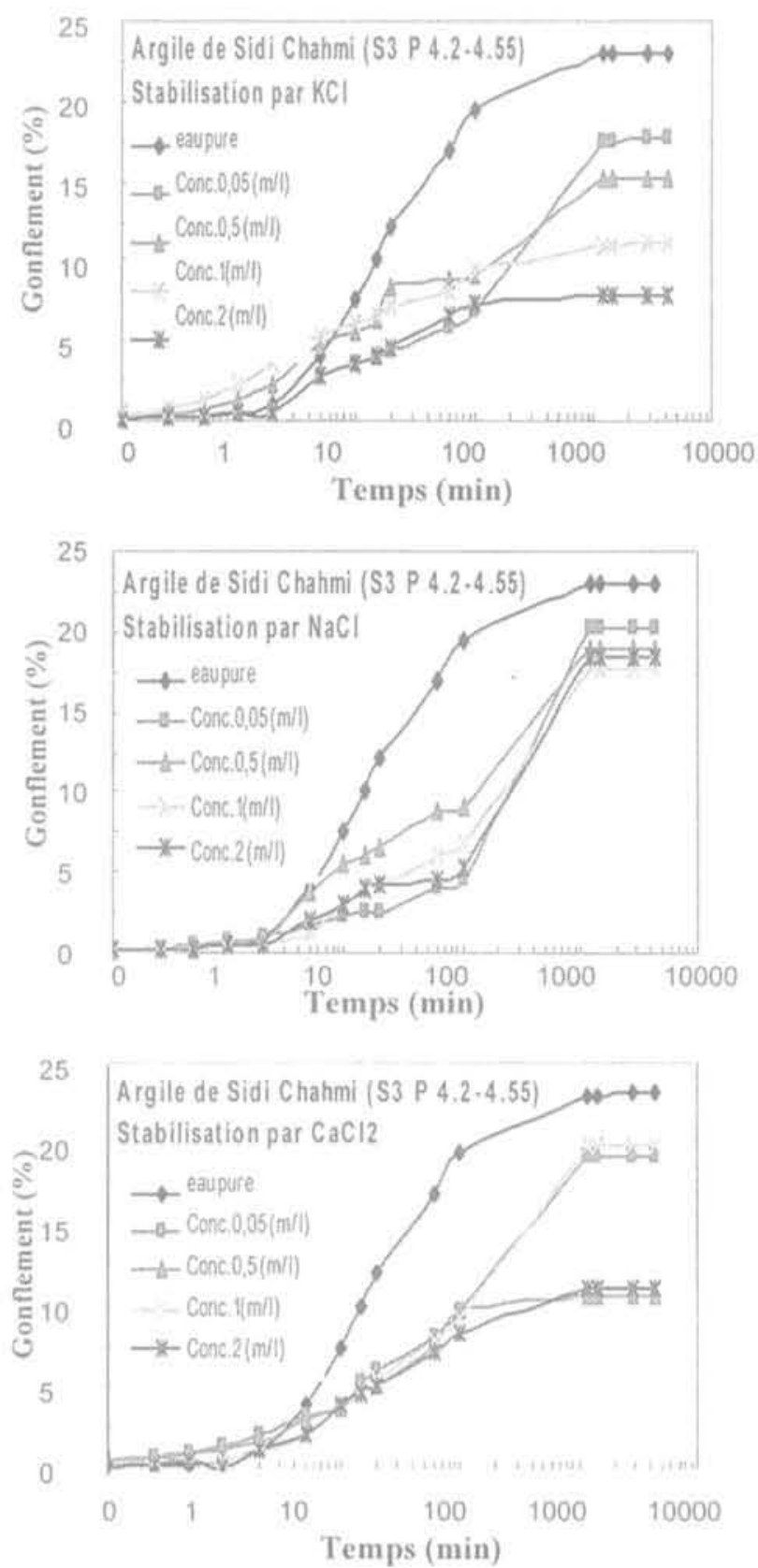

Le chlorure de sodium à forte concentration ne donne pas de bons résultats ( $22 \%$ et $28 \%$ ) pour l'argile de Sidi Chahmi et pour celle de Mers-el Kébir, respectivement, mais il a l'avantage d'être moins coûteux.

\section{2}

\section{Réduction du gonflement par les polymères}

Les courbes de gonflement des argiles de Sidi Chahmi et de Mers-el Kébir en présence des polymères qui ont donné le plus grand pourcentage de réduction sont représentées sur la figure 8 . On observe qu'au début des essais (environ $30 \mathrm{~min}$ ), le gonflement de l'argile en présence de polymères est plus élevé que celui en présence d'eau; ce qui est dû à l'accélération du gonflement par les polymères ; mais avec le temps, ce gonflement diminue. D'une manière générale, on
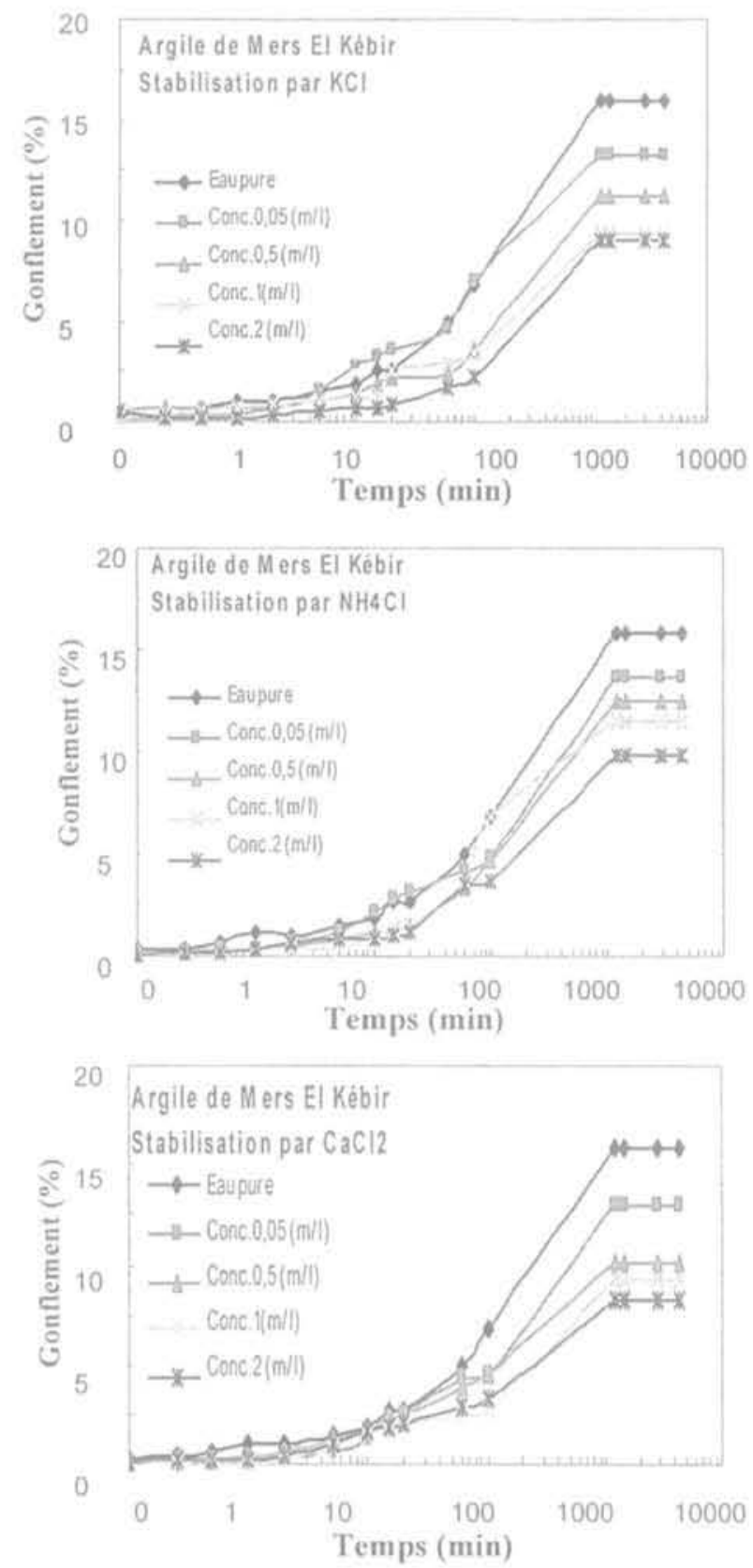

FlG.7 Courbes de gonflement des argiles de Sidi Chahmi et Mers-el Kébir en présence de différents sels minéraux à différentes concentrations.

Swelling curves of the clays treated with different mineral salts at different concentrations. 

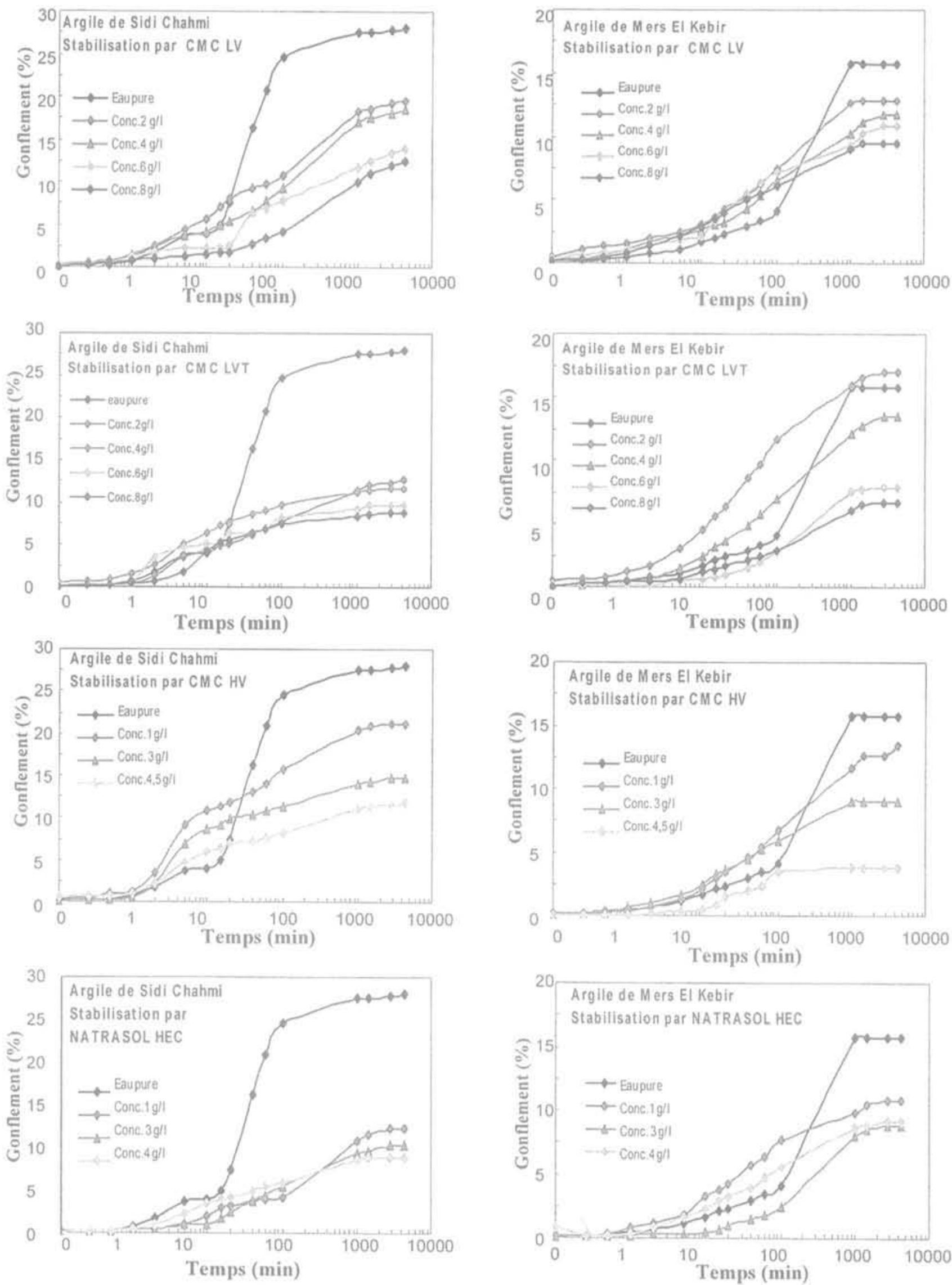

FIG. 8 Courbes de gonflement des argiles de Sidi Chahmi et Mers-el Kébir en présence de différents polymères à différentes concentrations.

Swelling curves of the clays treated with different polymers at different concentrations. 
remarque qu'une forte concentration en polymères a pour effet de diminuer le gonflement final. Les résultats obtenus montrent que ces sels organiques sont aptes à inhiber le gonflement des deux argiles. Nous pouvons retenir que le CMC HV à une concentration de $4,5 \mathrm{~g} / 1$ réduit considérablement (de $76 \%$ ) le gonflement de l'arcile de Mers-el Kébir. Le CMC LVT et le Natrasol HEC, à des concentrations de $8 \mathrm{~g} / \mathrm{l}$ et $4 \mathrm{~g} / \mathrm{l}$. respectivement, ont réduit chacun de $68 \%$ le gonflement de l'argile de Sidi Chahmi.

\section{3}

\section{Réduction du gonflement par l'association sels minéraux + polymères}

Les concentrations de sels et de polymères qui ont été choisies pour ces essais sont celles qui ont donné, quand les produits étaient utilisés seuls, les réductions de gonflement les plus fortes. Les sels utilisés sont le $\mathrm{KCl}$ à 2 moles/l, le $\mathrm{NaCl}$ à 1 mole/l, le $\mathrm{NH}_{4} \mathrm{Cl}$ à 2 moles $/ 1$ et le $\mathrm{CaCl}_{2}$ à 0,5 mole/l pour l'argile de Sidi Chahmi et 2 moles/ 1 pour l'argile de Mers-el Kébir. Les polymères choisis sont le CMC LV (à $8 \mathrm{~g} / 1$ ), le CMC LVT (à $8 \mathrm{~g} /$ ), le CMC HV (à $4,5 \mathrm{~g} / 1)$ et le NATRASOL HEVC (à $4 \mathrm{~g} / \mathrm{l})$.

L'effet des systèmes sels + polymères sur le gonflement final des argiles étudiées est indiqué dans le tableau III. On constate que les associations (sels + polymères) réduisent considérablement le gonflement final. Ces diminutions sont plus importantes que celles résultant de l'action du sel seul ou que celles données par une forte concentration des polymères. L'association du $\mathrm{NH}_{4} \mathrm{CL}$ et du CMC HV conduit à un gonflement final de l'ordre de $3 \%$ pour l'argile de Sidi Chahmi et de l'ordre de 3,4\% pour l'argile de Mers-el Kébir, soit des réductions d'environ $90 \%$ et $78 \%$, respectivement.

\section{7}

\section{Influence des additifs sels + polymères sur les limites d'Atterberg}

Afin d'étudier l'influence de la stabilisation sur les limites de consistance des deux argiles, des mesures des limites de liquidité, de plasticité et de retrait ont été effectuées avec les différents fluides hydratants. Les résultats de ces essais sont consignés dans le tableau IV. On constate que :

- dans la plupart des cas (sauf avec $\mathrm{CaCl}_{2}$ ), il se produit une diminution sensible de l'indice de plasticité de l'argile de Sidi Chahmi. Cette diminution est particulièrement sensible avec $\mathrm{KCl}+\mathrm{CMC}$ où la valeur passe de 60 à environ 15. Dans le cas du matériau de Mers-el Kébir, ce n'est qu'avec $\mathrm{KCl}$ et certains mélanges avec $\mathrm{NH}_{4} \mathrm{Cl}$ que l'on observe cette diminution. La diminution de $\mathrm{l}_{\mathrm{p}}$ est due le plus souvent à une augmentation de $\mathrm{w}_{\mathrm{p}}$, beaucoup plus qu'à une diminution de $\mathrm{w}_{1}$ que l'on n'observe qu'avec $\mathrm{KCl}$. En fait, le chlorure de potassium a un effet extrêmement positif, particulièrement en association avec la CMC car il conduit à une diminution sensible de la plasticité du sol ;

- dans le cas de l'argile de Sidi Chahmi, les résultats obtenus avec le chlorure de calcium sont très mauvais. On observe une augmentation de l'indice de plasticité qui résulte d'une forte augmentation de la limite de liquidité et d'une quasi-invariance de la limite de plasticité. Dans le cas de l'argile de Mers-el Kébir, le chlorure de calcium n'a pratiquement aucun effet ;

- avec le chlorure de sodium et le chlorure d'ammonium, les résultats sont plus mitigés et dépendent fortement de l'argile et du sel organique auquel ils sont associés. Pour l'argile de Sidi Chahmi, on observe une légère diminution de l'indice de plasticité (surtout avec $\mathrm{NH}_{4} \mathrm{Cl}$ ) due à une augmentation de la limite de plasticité et à l'invariance de la limite de liquidité. Avec l'argile de Mers-el Kébir, l'indice de plasticité augmente presque toujours (sauf avec $\mathrm{NH}_{4} \mathrm{Cl}+\mathrm{CMC} \mathrm{LV} \mathrm{ou} \mathrm{LVT),} \mathrm{avec} \mathrm{une}$ augmentation de $w_{\mathrm{L}}$ supérieure à l'augmentation de $\mathrm{w}_{\mathrm{p}}$.

En résumé, on peut dire que l'addition des différents sels et polymères entraîne souvent une diminution de la plasticité du matériau dans le cas de $\mathrm{KCl}$, une augmentation dans le cas de $\mathrm{CaCl}_{2}$ et des résultats moins nets pour les systèmes avec $\mathrm{NaCl}_{2}$ ou $\mathrm{NH}_{4} \mathrm{Cl}$. On observe donc une bonne corrélation entre ces résultats et ceux des essais de gonflement libre,

L'effet des additifs (polymère + sel) se traduit par une augmentation de l'indice de retrait $I_{\mathrm{H}}$. Des réductions de cet indice ont été obtenues avec le système $(\mathrm{KCl}+$ polymères) pour l'argile de Sidi Chahmi et par les associations $(\mathrm{KCl}+\mathrm{CMC} \mathrm{LV})$ et $(\mathrm{KCl}+\mathrm{HEC})$ pour l'argile de Mers-el Kébir.

TABLEAUIII Gonflement final et variation relative de gonflement pour les deux argiles traitées au sel + polymère. Final swelling and swelling relative change for the two clays treated with sait and polymer.

\begin{tabular}{|c|c|c|c|c|c|}
\hline \multirow[b]{2}{*}{$\mathrm{H}_{2} \mathrm{O}$} & & \multicolumn{2}{|c|}{$\begin{array}{l}\text { Sidi Chahmi } \\
\qquad G / G(\%)\end{array}$} & \multicolumn{2}{|c|}{ Mers-el Kébir } \\
\hline & & 28 & 0 & 15,8 & 0 \\
\hline $\mathrm{KCl}+$ & $\begin{array}{c}\text { CMCLVT } \\
\text { CMC LV } \\
\text { CMCHV } \\
\text { HEC }\end{array}$ & $\begin{array}{l}10,4 \\
10,6 \\
9,6 \\
7,7 \\
\end{array}$ & $\begin{array}{l}63 \\
64 \\
66 \\
73 \\
\end{array}$ & $\begin{array}{l}6,5 \\
6,2 \\
4,2 \\
4,9\end{array}$ & $\begin{array}{l}59 \\
61 \\
73 \\
69 \\
\end{array}$ \\
\hline $\mathrm{NaCl}+$ & $\begin{array}{c}\text { CMC LVT } \\
\text { CMC LV } \\
\text { CMCHV } \\
\text { HEC }\end{array}$ & $\begin{array}{l}12,5 \\
13,2 \\
13,4 \\
8,2 \\
\end{array}$ & $\begin{array}{c}55 \\
3 \\
52 \\
71 \\
\end{array}$ & $\begin{array}{l}6,5 \\
6,5 \\
4,2 \\
4,9\end{array}$ & $\begin{array}{l}59 \\
59 \\
57 \\
52 \\
\end{array}$ \\
\hline $\mathrm{NH}_{4} \mathrm{Cl}+$ & $\begin{array}{l}\text { CMCLVT } \\
\text { CMCLV } \\
\text { CMC HV } \\
\text { HEC }\end{array}$ & $\begin{array}{l}7,9 \\
9,2 \\
2,9 \\
3,8 \\
\end{array}$ & $\begin{array}{l}72 \\
67 \\
90 \\
86 \\
\end{array}$ & $\begin{array}{l}5,5 \\
5,6 \\
3,4 \\
4,4 \\
\end{array}$ & $\begin{array}{l}65 \\
64 \\
78 \\
72 \\
\end{array}$ \\
\hline $\mathrm{CaCl}_{2}+$ & $\begin{array}{l}\text { CMC LVT } \\
\text { CMC LV } \\
\text { CMC HV } \\
\text { HEC }\end{array}$ & $\begin{array}{l}18,5 \\
16,3 \\
15,7 \\
11,4\end{array}$ & $\begin{array}{l}34 \\
42 \\
44 \\
59\end{array}$ & $\begin{array}{l}8,6 \\
6,8 \\
6,9 \\
8,2\end{array}$ & $\begin{array}{l}45 \\
57 \\
56 \\
48\end{array}$ \\
\hline
\end{tabular}


L'association des sels et des polymères conduit à une diminution importante de la limite de retrait des deux argiles, à l'exception des systèmes ( $\mathrm{NaCl}+\mathrm{HEC}$ ) et $(\mathrm{KCl}+\mathrm{HEC})$ où la limite de retrait a considérablement augmenté pour l'argile de Mers-el Kébir. La limite de retrait de l'argile Sidi Chahmi a subi une augmentation non négligeable dans le cas du système $(\mathrm{KCL}+\mathrm{HEC})$. Ces variations de la limite de retrait traduisent la diminution du potentiel de gonflement des deux arciles.

\section{8}

\section{Conclusion}

Le gonflement des sols est un phénomène très complexe qui fait intervenir un grand nombre de paramètres intrinsèques et environnementaux. Lors de cette étude, nous avons essayé de trouver une solution afin d'éviter que le gonflement ne se produise sous des ouvrages en procédant par une stabilisation des sols avant les travaux de fondation. Cette stabilisation peut se faire en injectant des solutions salines qui ont montré leur efficacité dans la réduction du gonflement. Afin de vérifier l'efficacité des solutions en place, il faut prélever des échantillons intacts avant et après traitement et mesurer leur gonflement au laboratoire.

L'application de la technique de stabilisation des sols gonflants par les sels, les polymères ou la combinaison des deux à des ouvrages réels doit être envisagée avec prudence car il n'est pas impossible que les sels soient lessivés par l'eau et que leur effet finisse par disparaitre. Pour cela, il faudrait mener une étude spécifique détaillée de lixiviation, si possible dans les conditions du site, avant chaque cas de traitement, afin de définir la nature et la concentration des solutions salines à utiliser.

L'étude de l'influence du fluide hydratant sur les limites d'Atterberg des deux argiles a mis en évidence une bonne corrélation entre les diminutions de l'indice de plasticité obtenues avec différents systèmes (selpolymère) et les réductions de gonflement mesurées en présence de ces systèmes. Des réductions importantes de l'indice de plasticité et de retrait ont été obtenues en particulier avec le chlorure de potassium ; en revanche; lutilisation du chlorure de calcium a conduit, dans la plupart des cas, à une augmentation de l'indice de plasticité et dé la limite de liquidité.

TABIEAU IV Limites d'Atterberg et de retrait mesurées en présence de différents additifs sels/polymères. Atterberg and shrinkage limits of the two clays with different salt-polymer additives.

\begin{tabular}{|c|c|c|c|c|c|c|c|c|c|c|c|c|c|c|c|c|c|c|}
\hline & & & & 100 & $M / 1)$ & & & $\mathrm{KC}$ & & & & $\mathrm{NH}_{4} \mathrm{Cl}$ & $2 \mathrm{M} / \mathrm{l})$ & & & $\mathrm{CaCL}_{2}(\mathrm{l}$ & $5 \mathrm{M} / \mathrm{l})$ & \\
\hline & & 10 & $\begin{array}{l}\text { CMC } \\
\text { LVI } \\
(8 \mathrm{~g} / 1)\end{array}$ & $\begin{array}{l}\text { CMC } \\
L V \\
(8 \mathrm{~g} / \mathrm{A})\end{array}$ & $\begin{array}{c}\text { CMC } \\
\mathrm{HV} \\
(4,5 \mathrm{~g} / 1)\end{array}$ & $\begin{array}{l}\text { HEC } \\
(4 g / 1)\end{array}$ & $\begin{array}{l}\mathrm{CMC} \\
\mathrm{LVT} \\
(8 \mathrm{~g} / \mathrm{l})\end{array}$ & $\begin{array}{c}\text { CMC } \\
L V \\
(8 g / 1)\end{array}$ & $\begin{array}{c}\text { CMC } \\
H V \\
(4,5 g / 1)\end{array}$ & $\begin{array}{l}\text { HEC } \\
(4 g / 1)\end{array}$ & $\begin{array}{l}\mathrm{CMC} \\
\mathrm{LVT} \\
(8 \mathrm{~g} / 1)\end{array}$ & $\begin{array}{c}\text { CMC } \\
\text { LV } \\
(8 g /)\end{array}$ & $\begin{array}{c}\text { CMC } \\
\text { HV } \\
(4,5 \mathrm{~g} / 4)\end{array}$ & $\begin{array}{l}\text { HEC } \\
(4 g / 1)\end{array}$ & $\begin{array}{l}\text { CMC } \\
\text { LVT } \\
(8 \mathrm{~g} / 1)\end{array}$ & $\begin{array}{c}\text { CMC } \\
\mathrm{LV} \\
(8 \mathrm{~g} / 1\end{array}$ & $\begin{array}{c}\text { CMC } \\
\text { HV } \\
(4.5 \mathrm{~g} /)\end{array}$ & $\begin{array}{l}\text { HEC } \\
(4 \mathrm{~g} / \mathrm{d})\end{array}$ \\
\hline & u & & 83,8 & 88,4 & 77.6 & 86,6 & 62,2 & 64 & 56,2 & 83,0 & 8 & 77.7 & 89,2 & 83,0 & 99,5 & 102,8 & 93,6 & 113.6 \\
\hline & $w_{p}$ & 25,0 & 35,9 & 37,7 & 38,8 & 39,1 & 46,8 & 42 & 39,3 & 41,8 & & 45,0 & 44,9 & 46.3 & 2 & 32.9 & 6 & 33,4 \\
\hline & $w_{\mathrm{h}}$ & 8,0 & 6,7 & 6,6 & 4,0 & 4,53 & 5,8 & 5,13 & 5,1 & & & & & & & & & 7,1 \\
\hline & $I_{p}$ & 48,8 & 471 & 50,6 & 38,7 & 47.5 & 154 & 22,04 & 160 & 4 & 38,1 & 3 & 44,3 & 30 & 6 & 69,8 & 1 & 80,2 \\
\hline & $I_{A}$ & 65,0 & 7,1 & 81,8 & 730 & 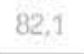 & & $=0$ & 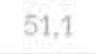 & $=$ & & & & & & & 0 & 106,5 \\
\hline & w & 49,0 & . & 02,0 & 列 & 然 & 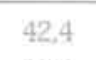 & 46 & $4=$ & ${ }^{4}$ & & & & & & & & 48,0 \\
\hline & $w_{p}$ & 24,0 & 24.7 & 25,7 & 31,7 & 26,6 & 2 & 30 & 26.6 & & & & & & & & & 19,2 \\
\hline- & $w_{n}$ & 12,0 & 8,8 & 6,5 & 11.4 & 23,7 & 3,2 & 14.7 & 3,8 & 26,0 & 7,2 & 4,2 & 2.0 & 7.6 & 47 & 3. & 10.6 & 5,0 \\
\hline & $I_{p}^{\prime \prime}$ & 26.0 & 39,2 & 37.8 & 30,0 & 36.1 & 14,9 & 16, & 17,8 & 19 & 23,3 & 22,5 & 40,7 & 43,9 & & art & & 29,8 \\
\hline & $I_{R}$ & 37,0 & 54,6 & 56,3 & 50,3 & 39,9 & 39,2 & 32,0 & 40,6 & 21,7 & 46,7 & 47,3 & 62,2 & 68.5 & 37.7 & 40,2 & 36,5 & 44,0 \\
\hline
\end{tabular}

\section{Bibliographie}

Ameur B. - Sols gonflants : étude de cas Comptes rendus du Symp. Int de Mécranique des Sols de Tiaret (Alqérie). CORT-LTPO (èd.), juin 1989, tome 1.

Azzouz S.R. - Contribution à l'étude de la stabilité des argiles feuilletées en cours de forace. Thèse de docteur incénieu de l'Institut national des sciences appliquées de Lyon, 1983.

Basma A.A. Al-Rawas A.A. Al-Saadi SN. Al-Zadjali I.F. - Stabilisation of expansive clays in Oman. Environmental and Engineering Geoscience, 4 (4), 1998, p. 503-510.

Bourokba A.S., Hachichi A., Benaissa A. 2001. Étude du gonflement et de la stabilisation chimique de quelques sols gonflants de la région d'Oran. Comptes rendus du Séminaire national de génie civil. Sidi-bel-Abbès, Algérie, 2001, tome 2

Breen C. - The characterisation and use of polycation-exchanged bentonites. Applied Clay Science, 15, 1999, p. 187-219.

Chaouch Y. - Contribution à l'étude du comportement hydromecanique des sols gonflant. These de doctorat en sciences appliquées, Universitè libre de Bruxelles, septembre 2001.

Derrich Z., Iguechtal L., Tas M. - Comportement des ouvrages dans les arqiles expansives d'In-Aménas. Revue francaist de géotechnique, $n^{\circ} 89,1999$, p. 55-65. Hachichi A. Fleureau J.-M - Caractérisa. tion et stabilisation de quelques sols gonflants d'Algèrie. Revue française de géotechnique, $n^{=} 86,1999$, p. 37-51.

Hachichi A. Bourokba A.S. Boudia M. Etude de la stabilisation par polymères de quelques sols gonflants de la régior d'Oran. Comptes rendus de la Conférence internationale de la construction urbaine. Université des sciences et de la technologie Houari Boumédienne, Alger, juin 2002.

Ho Young J. Takeshi K., Craig H.B. Tuncer B.E. - Hyciraulic conductivity and swelling of nonprehydrated GCLs permeated by single-species salt solu- tions. Journal of Geotechnical and Geoenvironmental Engineering, ASCE. 127 (7). 2001, p. 557-557

Lamara M Derrich Z Romero F - Case study of swelling induced ctamage and characterisation of an arid climate soil. Proc. Int. Conf on Problematic Soils. Eastern Mediterranean University, Famagusta, Cyprus, May 2005, p. 25-27.

Suratman 1. - Contribution à l'étude de la cinétique et de la stabilisation du gonflement des argiles. Thése de docteur ingé. nieur de l'Institut national des sciences appliquées de Lyon, 1985.

Tran N. L. - Nouvel essai d'identification des sols : l'essai au bleu de méthylène. Bulletin de liaison des laboratoires des ponts et chauscees, 88, mars-avril 1977. p. 136-137.

Williams A.B., Donaldson G.W.-Developments related to building on expansive soils in South Africa: 1973-1980. Proc 4th. Int. Conf on Expansive Soils, Denver, vol. 2, 1980, p. 834-844. 